- This study highlights the fact that some commercially available mouthrinses may not achieve the plaque inhibitory activity that they are expected to have.

- Ingredients contained within some cationic antiseptic mouthrinse solutions may inactivate the plaque inhibitory agent.

- The study confirms the varying ability of different mouthrinses to form stains in the laboratory and this has been found in many previous studies to be indicative of clinical staining and plaque inhibitory efficacy.

\title{
An in vitro evaluation of the availability of cetylpyridinium chloride and chlorhexidine in some commercially available mouthrinse products
}

\author{
S. Sheen ${ }^{1}$ and M. Addy ${ }^{2}$
}

Objectives To assess the comparative activity of mouthrinses containing cetylpyridinium chloride (CPC) and chlorhexidine using the propensity to cause extrinsic staining in vitro as the outcome variable. Methods Saliva-coated clear acrylic specimens were exposed to cyclical staining regimens of either CPC or chlorhexidine followed by tea. Water and $0.2 \%$ chlorhexidine were used as negative and positive controls respectively. Staining cycles were repeated until an optical density of $>2$ was reached by one of the products.

Results For CPC there was a highly significant difference in staining between the products. Two CPC products performed numerically little better than water. For the chlorhexidine products the $0.2 \%$ formulation produced the most staining although little more than the UK version of the $0.1 \%$ rinse. The French $0.1 \%$ rinse produced by the same manufacturer as the UK formulation showed markedly reduced staining potential although significantly greater than water.

Conclusion This study, supported by previous in vitro and in vivo studies, indicates discrepancies in the availability of CPC and chlorhexidine in some mouthrinse products. Importantly, this may have an effect on the potential of some rinses to provide the expected plaque inhibitory activity.

Mouthrinses have been used by populations throughout the world for centuries. Indeed, the first reference to mouthrinsing as a formal practice can be found in Chinese medicine and dates back as far as 2700 вс. ${ }^{1}$ Traditionally mouthrinses were thought to provide oral health benefits and were also used cosmetically as a way of freshening breath. ${ }^{2}$ However, it is now known that mechanical

${ }^{1}$ Lecturer, ${ }^{2 *}$ Professor, Division of Restorative Dentistry, Dental School, Bristol Correspondence to: Prof. Martin Addy, Division of Restorative Dentistry, Bristol Dental School, Lower Maudlin Street, Bristol BS1 2LY

E-mail: Martin.Addy@bristol.ac.uk

\section{Refereed paper}

Received 26.06.01; Accepted 23.09.02

(๑) British Dental Journal 2003; 194: 207-210 cleaning alone rarely leads to a plaque-free environment ${ }^{3,4}$ and therefore mouthrinses have been used more recently as vehicles for plaque inhibitory agents directed at improving plaque control and or gingival health (for review see references 5 - 7). A wide variety of mouthrinse products are freely available 'over the counter' to the consumer today and figures would indicate that they are commonly used in the home, with the sale of mouthrinses in the UK in 1999 reaching £73 million.

Among the available mouthrinses, chlorhexidine formulations remain undisputed as the gold standard among anti-plaque mouthrinses, ${ }^{8,9}$ but local side effects have tended to restrict their use to the short to medium term. The most significant local side effect of chlorhexidine is the well known formation of extrinsic dental staining of teeth, oral mucosa, restorative materials and acrylic dentures. ${ }^{10,11}$ Other readily available mouthrinses are perhaps less well known for their undesirable staining capabilities. For example, cetylpyridinium chloride (CPC), which is perhaps the most common ingredient in over-the-counter products, can produce dental staining ${ }^{12,13}$ although the degree of staining formed has been noted to be considerably less than that of chlorhexidine. ${ }^{14}$ In addition staining by essential oil mouthrinses has been noted. ${ }^{15}$ The effect of stain removal by the mechanical action of brushing when mouthrinses are used as adjuncts to brushing may be one explanation for the apparently infrequently noted clinical staining caused by these other rinses.

Several mechanisms have been proposed for the aetiology of extrinsic dental staining associated with cationic antiseptics and some polyvalent metal salts (for review see reference 16). However, both laboratory and clinical evidence strongly support a dietary aetiology whereby staining is caused by the precipitation of dietary chromogens by cationic antiseptics such as chlorhexidine and CPC. ${ }^{16-20}$ This phenomenon has been exploited in vitro to assess the availability of cationic antiseptics in commercially available mouthrinse formulations. ${ }^{21-23}$ Indeed, the method has been employed to predict plaque inhibitory properties of mouthrinses containing cationic antiseptics. ${ }^{22,24-28}$ 
The aim of this study was to compare the in vitro staining capability and hence the availability of CPC and chlorhexidine in various brands of commercially available mouthrinse solutions. It was then hoped that the results would allow the possible prediction of in vivo activity among products containing the same antiseptic to be made.

\section{MATERIALS AND METHODS}

The method involved cationic antiseptic enhanced tea staining of optically clear methyl methacrylate specimens (Perspex. Amari Plastics plc., Weybridge, Surrey). Rectangular specimens measuring $30 \times 10 \times 5 \mathrm{~mm}$ were prepared to fit the specimen chamber of a UV/visible spectrophotometer. Six specimens were used per experimental group and for logistical purposes it was necessary to run two separate studies under identical laboratory conditions. Specimens were baselined at the lambda maximum for tea namely $295 \mathrm{~nm}$ on a UV/visible double beam spectrophotometer. Specimens were then soaked in human saliva for 2 minutes, washed in distilled water and immersed for 2 minutes in a $10 \mathrm{ml}$ solution of each of the 14 chosen mouthrinse solutions (see Table 1). A $0.2 \%$ chlorhexidine rinse and distilled water were used in both experiments to act as positive and negative standards respectively for each experiment. This would allow observational comparisons to be made between the two experiments on products containing CPC.

Following exposure to the different solutions, specimens were removed, washed in distilled water, and exposed to a $10 \mathrm{ml} \mathrm{stan-}$ dard tea solution for 1 hour. The standard tea solution was prepared by using $1 \mathrm{~g}$ of tea leaves per $100 \mathrm{ml}$ of boiled tap water. The solution was left to stand for 3 minutes, strained through gauze and left to cool to room temperature. At the end of 1 hour, specimens were removed, washed in distilled water and left to dry before reading on the spectrophotometer. This process was then repeated until the average optical density reading of six blocks of one or more groups at that cycle reached a value of $>2.0$.

\section{STATISTICAL METHODS}

In this method, to compare activity, comparisons can only be made between the negative standard water and products with the same ingredients, ie CPC or chlorhexidine. Dentyl pH had a noticeable effect on the property of the specimen surfaces and although staining was seen, the optical density readings were considered to be invalid. For the first study, in order to avoid multiple comparisons, one-way analysis of variances were per- formed on the CPC containing rinses (excluding Dentyl $\mathrm{pH}$ for reasons mentioned above) with and without water. If statistically significant differences were found, the pre-study plan was to perform an unpaired $t$ test between the least staining CPC rinse and water and the next least staining CPC rinse at the 5\% level of significance. Alpha errors were minimised by using a Bonferroni correction. Thus, statistical analysis was only performed between Boots Total Care and water as no significant differences were found between the five CPC rinses without water. For the second study involving six CPC rinses and water the planned statistical analysis was as for study 1 . For the chlorhexidine rinses again analysis of variances were performed between the three rinses with and without water, if significant, it was planned to compare the lowest staining rinse with water and the next least staining chlorhexidine rinse using unpaired $t$ tests with a Bonferroni correction.

\section{RESULTS}

In both studies a total of six staining cycles were required before an average optical density of $>2.0$ was produced, and this was in the $0.2 \%$ chlorhexidine groups (positive control). The results for each group at cycle 6 can be seen in Figures 1 and 2. In both experiments, $0.2 \%$ chlorhexidine rinse stained the most and to a

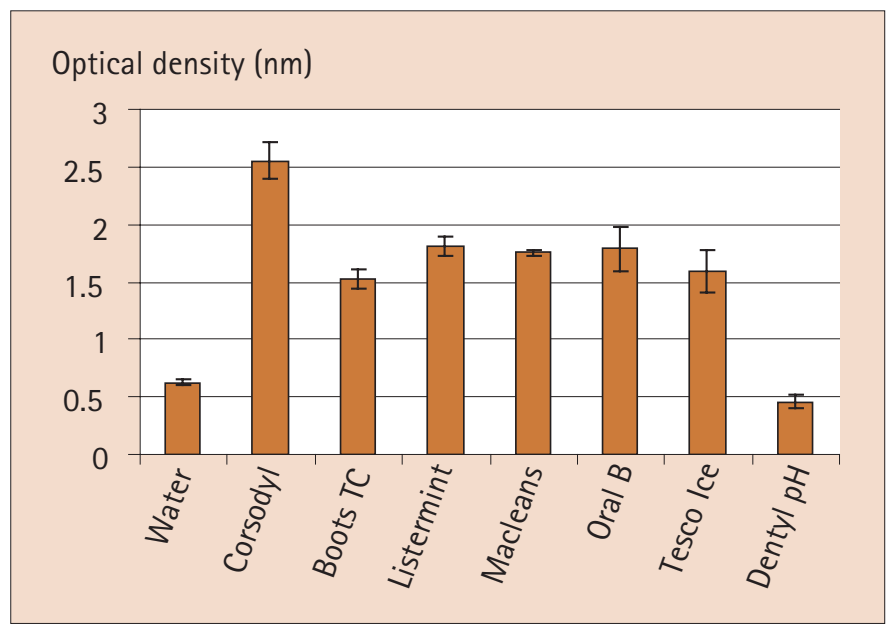

Fig. 1 Mean extrinsic stain formation with standard error bars at cycle 6 -study 1

\begin{tabular}{l|l|c}
\hline \multicolumn{2}{l}{ Table 1 Formulations used in the two stain formation studies. } & \\
\hline Formulation & Manufacturer/Retailer & Study \\
\hline Boots Total Care & The Boots Company PLC, Nottingham & 1 \\
\hline Listermint & Warner-Lambert Consumer Healthcare, Eastleigh, Hampshire, S053 3ZO. & 1 \\
\hline Macleans Mouthguard & SmithKline Beecham Consumer Healthcare, Brentford, TW8 9BD & 1 \\
\hline $\begin{array}{l}\text { Oral B Antiplaque } \\
\text { Alcohol Free Mouthwash }\end{array}$ & Oral-B Laboratories, Ireland, Newbridge, Co. Kildare & 1 \\
\hline Tesco Icemint & Tesco Stores Ltd, Cheshunt, EN8 9SL. & 1 \\
\hline Dentyl pH & Fresh Breath Ltd, Conan Doyle House, 2 Devonshire Place, London W1N 1PA & 1 \\
\hline $\begin{array}{l}\text { Superdrug Just Great } \\
\text { Value Mouthwash }\end{array}$ & Superdrug Stores PLC, Admail 838, Croydon, CR9 4WZ & 2 \\
\hline Superdrug Total Care & Superdrug Stores PLC, Admail 838, Croydon, CR9 4WZ \\
\hline Freshmint Mouthwash & Sainsbury's Supermarkets Ltd, Stamford Street, London SE1 9LL \\
\hline $\begin{array}{l}\text { Sainsbury's Extra Strength } \\
\text { Antiseptic Mouthwash }\end{array}$ & Sainsbury's Supermarkets Ltd, Stamford Street, London SE1 9LL \\
\hline Sainsbury's Freshmint Mouthwash & Carter-Wallace Ltd, Folkestone, Kent & 2 \\
\hline Pearl Drops Smokers 1+1 Mouthwash & Procter \& Gamble, Cincinnati, Ohio 45202, USA & 2 \\
\hline Scope Original Mint Mouthwash & Pierre Fabre Ltd, Hyde Abbey House, 23 Hyde Street, Winchester, Hants SO23 7DR & 2 \\
\hline Eludril Mouthwash & Pierre Fabre Medicament, 45 Place Abel-Gance, 92100 Boulogne, France & 2 \\
\hline Solution Eludril & & 2 \\
\hline
\end{tabular}




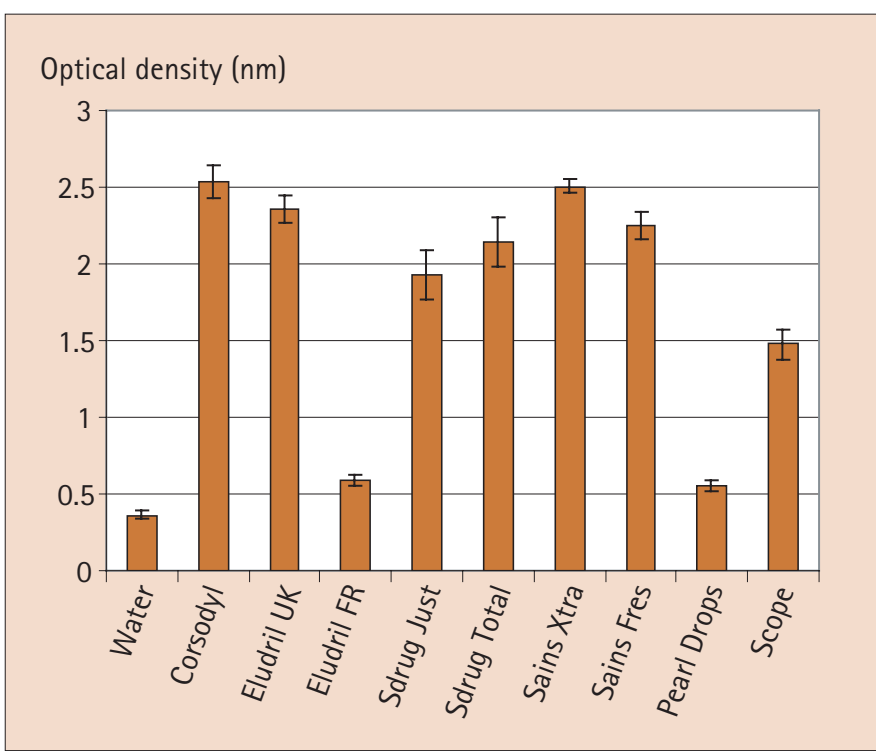

Fig. 2 Mean extrinsic stain formation with standard error bars at cycle 6 study 2

very similar level of optical density. The negative standard water produced little staining but the optical density was lower in the second compared with the first study. Across studies therefore it is apparent that there was considerable variation in staining potential of the CPC rinses. Thus two products produced staining at or around an optical density of 0.5 , and therefore similar to water, seven products achieved an optical density of around 1.5 to just $<2.0$ and three products exceeded an optical density of 2.0. For the chlorhexidine rinses in the second study the $0.2 \%$ and UK $0.1 \%$ chlorhexidine products achieved an optical density respectively of just greater than and just less than 2.5 , but the equivalent French version of $0.1 \%$ chlorhexidine achieved an optical density of around 0.5.

In study 1 (Fig. 1), analysis of variance excepting the data for Dentyl pH showed no significant difference between the other 5 CPC rinse products without water $(P=0.473)$. However, with water the difference was extremely significant $(P<0.001)$. The difference between Boots Total Care (the CPC with the lowest optical density of the 5 rinses) and water was found to be highly significant $(P<0.001)$ with a difference in mean staining at cycle 6 of $59 \%$.

In study 2 (Fig. 2), analysis of variance across the six CPC rinse products without water revealed highly significant differences between products $(P<0.001)$. This difference appeared largely due to Pearl Drops Smokers which achieved the lowest optical density of all the products in this experiment and to a level highly significantly lower than the rinse with the next lowest optical density, namely Scope $(P<0.001)$ there being 63\% more stain formed with Scope. An analysis of variance with water showed highly significant differences across the groups $(P<0.001)$. Despite the much reduced staining by Pearl Drops Smokers, a 35\% increase in staining was seen compared with water which was significantly greater $(P=0.002)$.

Stain formation amongst the chlorhexidine containing rinses both with and without water in study 2 was found to differ to a highly significant degree $(P<0.001)$ with the most stain at cycle 6 being formed by the $0.2 \%$ product, followed by the UK and finally the French $0.1 \%$ chlorhexidine formulations. The UK formulation produced almost four times the amount of stain compared with the French formulation, a difference which was highly significant $(P<0.001)$. Compared with water, the increase in stain produced by the French formulation was numerically small but highly significant $(P<0.001)$.

\section{DISCUSSION}

The use of optically clear acrylic specimens to assess and measure in vitro staining has been used on many occasions in previous studies. ${ }^{18,29-31}$ This study involved the pre-treatment of such specimens with saliva as this is known to significantly increase in vitro staining. ${ }^{32}$ The optical density of stain produced on the specimens throughout progressive cycles was used to assess the availability of cationic antiseptics within several mouthrinse solutions. A total of twelve CPC rinses were examined. A concentration of $0.05 \%$ CPC was stipulated in only two of these formulations. However, this is the most common concentration known to be incorporated in commercial formulations and CPC has been found to be most effective in plaque re-growth studies at concentrations of between $0.05 \%$ and $0.1 \%$ (for review see reference 6).

This study complemented the findings of other studies in the following respects. Firstly, in a study comparing in vitro staining by various CPC rinses, ${ }^{21}$ a Sainsbury's formulation performed exceptionally well. In the same study, a UK formulation containing $0.1 \%$ chlorhexidine was found to produce a comparable, if not greater amount of mean staining than a $0.2 \%$ chlorhexidine rinse, a finding which is not altogether surprising since the optimal concentration for chlorhexidine staining in vitro is known to peak at $0.1 \%{ }^{32}$ Secondly, staining by chlorhexidine and several of the CPC containing rinses was essentially similar. CPC has been shown to adsorb to acrylic to a greater extent than chlorhexidine, ${ }^{18,31}$ but the overall increased staining seen in the positive control groups using $0.2 \%$ chlorhexidine may be explained by the dicationic nature of chlorhexidine which may account for this difference once the antiseptic has been adsorbed.

The absence of dental staining in short term non-brushing clinical trials involving cationic antiseptics has been shown to be reflected in a lack of plaque inhibitory activity. ${ }^{26,33}$ This can be said either to be caused by the failure to comply with rinsing regimens on the part of the patient, or due to the active ingredient incorporated in the mouthrinse not being available. Indeed, research has confirmed that the mere incorporation of an active ingredient in a formulation does not necessarily imply clinical efficacy. ${ }^{24,27}$ Other studies in vitro and in vivo have consistently demonstrated that attempts to block staining by chlorhexidine and CPC result in a loss of plaque inhibitory activity. ${ }^{22,24}$ Although laboratory data clearly cannot stand alone, cautious extrapolation of activity to the clinical situation may be made between products containing the same active ingredient and one particular previous study has managed to position the clinical efficacy, in mean terms, for several chlorhexidine containing mouthrinses in order using in vitro staining data. ${ }^{24}$

Applying this knowledge to these in vitro studies, therefore, one may predict that there would be a decreased availability of CPC in several of the mouthrinses used in both experiments, and of a decreased availability of chlorhexidine in the French $0.1 \%$ chlorhexidine formulation compared with the UK version, as numerically, staining in this group was little different from water. These findings may in turn imply a decreased clinical efficacy although obviously, clinical studies are undoubtedly required to back up this conclusion. However, a previous clinical study on the French $0.1 \%$ chlorhexidine formulation ${ }^{24}$ found it to perform poorly both in terms of plaque inhibition and salivary bacterial counts compared with other chlorhexidine rinses. In addition to this, disappointing studies in vitro and in vivo of the original $0.1 \%$ chlorhexidine rinse available in the UK at the time ${ }^{26,34}$ led to a formulation change to improve activity. ${ }^{23}$ These findings clearly indicate that the manufacturers of the $0.1 \%$ chlorhexidine product reformulated in the UK but not in France. Researchers have proposed that the addition of certain ingredients such as detergents into mouthrinses may be responsible for the apparent lack of availability of some cationic antiseptics. For example, it is known 
that inactivation of cationic antiseptics may occur by anionic compounds such as sodium lauryl sulphate. ${ }^{35}$ Interestingly, Pearl Drops Smokers CPC mouthwash contains sodium lauroyl sarcosinate and this may perhaps, therefore, explain its poor staining performance which was only marginally greater than water.

Dentyl pH was not included in the statistical analysis of mean group staining as a sticky film was noted to form on the specimens in this group and this may have affected true optical density readings. Manufacturers' guidelines about this product recommend avoiding contact with plastics containing polystyrene, and this may have been the reasoning behind these findings.

In summary, this study has highlighted varying in vitro staining capabilities of commercially available mouthrinse products containing the same active ingredients. Previous research has demonstrated that staining by cationic antiseptics in vitro has correlated well with in vivo staining and plaque inhibitory activity. The study has also predicted a lack of availability of both CPC and chlorhexidine in some of the mouthrinses evaluated in this study in vitro. In conclusion, based on previous studies in vitro and in vivo, these experiments suggest that several CPC and chlorhexidine mouthrinses will not achieve the expected chemical plaque inhibitory benefits for which they were formulated.

1. Weinberger B. Introduction to the history of dentistry. St Louis: Mosby Co, 1948.

2. Golding PS. The development of the toothbrush. A short history of tooth cleansing Part 1. Dent Health 1982; 21: 25-27.

3. Alexander A G. A study of the distribution of supra and subgingival calculus, bacterial plaque and gingival inflammation in the mouths of 400 individuals. J Periodont 1971; 42: 21-28.

4. Addy M, Griffiths G, Dummer P M H, Kingdom A, Shaw W C. The distribution of plaque and gingivitis and the influence of toothbrushing hand in a group of South Wales 11-12 year-old children. J Clin Periodonto/ 1987: 14: 564-572.

5. Kornman KS. Antimicrobial agents. In: Löe H, Kleinman D W (eds), Dental plaque control measures and oral hygiene practices. Oxford: IRL press 1986; pp 121-142.

6. Mandel I D. Chemotherapeutic agents for controlling plaque and gingivitis. J Clin Periodontol 1988; 15: 488-496.

7. Gjermo P. Chlorhexidine and related compounds. J Dent Res 1989; 65: 1603-1608.

8. Addy M. Chlorhexidine compared with other locally delivered anti-microbials. A short review. J Clin Periodontol 1986: 13: 957-964.

9. Jones C G. Chlorhexidine: is it still the gold standard? Periodonto/2000 1997; 15: 55-62.

10. Flotra L. Different modes of chlorhexidine application and related local side effects. J Periodont Res 1973; 8(suppl 12): 41-44.

11. Olsen I. Denture stomatitis. The clinical effects of chlorhexidine and Amphotericin B. Acta Odontol Scand 1975; 33: 47-52

12. Ciancio S G, Mather BS, Burnell H L. The effect of a quaternary ammonium containing mouthwash on formed plaque. Pharmacol Ther Dent 1978; 3: 1-6.

13. Lobene R R, Kashket S, Soparker P M, Schloss J, Sabine Z M. The effect of CPC on human plaque bacteria and gingivitis. Pharmacol Ther Dent 1979: 4: 33-47.

14. Llewelyn J. A double blind cross-over trial on the effect of CPC $0.05 \%$ (Merocet) on plaque accumulation. Br Dent J1980; 148: 103-104.

15. Moran J, Pal D, Newcombe R G, Addy M. Comparison of phenolic and 0.2\% chlorhexidine mouthwash products on the development of plaque and gingivitis. Clin Prevent Dent 1991; 13: 31-34.

16. Addy M, Moran J. Mechanisms of stain formation on teeth, in particular associated with metal ions and antiseptics. Adv Dent Res 1995; 9: 450-456

17. Addy M, Roberts W R. Comparison of the bisbiguanide antiseptics alexidine and chlorhexidine. Il. Clinical and in vitro staining properties. J Clin Periodonto/ 1981; 8: 220-230.

18. Addy M, Moran J. The formation of stain on acrylic surfaces by the interaction of cationic antiseptic mouthwashes and tea. J Biomed Mat Res 1984; 18: 631-641.

19. Prayitno $S$, Taylor $L$, Cadogan $S$, Addy M. An in vivo study of dietary factors in the aetiology of tooth staining associated with the use of chlorhexidine. J Periodont Res 1979: 14: 411-417.

20. Addy M, Moran J, Griffiths A A, Wills-Wood N J. Extrinsic tooth discoloration by metals and chlorhexidine. I. Surface denaturation or dietary precipitation? Br Dent J 1985: 159: 281-285

21. Addy M, Mahdavi S A, Loyn T. Dietary staining in vitro by mouthrinses as a comparative measure of antiseptic activity and predictor of staining in vivo. J Dent 1995; 23: 95-99.

22. Addy M, Wade W G. An approach to efficacy screening of mouthrinses: studies on a group of French products (I). Staining and antimicrobial properties in vitro. J Clin Periodontol 1995; 22: 718-722.

23. Addy M, Wade W, Goodfield S. Staining and antimicrobial properties in vitro of some chlorhexidine formulations. Clin Prevent Dent 1991; 12: 13-17.

24. Harper PR, Milsom S, Wade W G, Addy M, Moran J, Newcombe R G. An approach to efficacy screening of mouthrinses: studies on a group of French products (II). Inhibition of salivary bacteria and plaque in vivo. J Clin Periodontol 1995; 22: 723 727.

25. Addy M, Moran J, Newcombe R G. A comparison of $0.12 \%$ and $0.1 \%$ chlorhexidine mouthrinses in the development of plaque and gingivitis. Clin Prevent Dent 1989; 13: 26-29.

26. Jenkins S, Addy M, Newcombe R G. Comparison of two commercially available chlorhexidine mouthrinses: II. Effects on plaque reformation, gingivitis and tooth staining. Clin Prevent Dent 1989; 11: 12-16.

27. Mendieta C, Vallcorba N, Binney A, Addy M. Comparison of 2 chlorhexidine mouthwashes on plaque regrowth in vivo and dietary staining in vitro. J Clin Periodontol 1994: 21: 296-300.

28. Smith R G, Moran J, Addy M, Doherty F, Newcombe R G. Comparative staining in vitro and plaque inhibitory properties in vivo of $0.12 \%$ and $0.2 \%$ chlorhexidine mouthrinses. J Clin Periodonto/ 1995; 22: 613-617.

29. Addy M, Roberts W R. The use of polymethylmethacrylate to compare the adsorption and staining reactions of some cationic antiseptics. J Periodont 1981b; 52: 380-385.

30. Moran J, Addy M. The effect of surface adsorption and staining reactions on the antimicrobial properties of some cationic antiseptic mouthwashes. J Periodont 1984; 55: $278-282$

31. Moran J, Addy M. The pattern of adsorption of cationic antiseptics to polymethylmethacrylate. J Oral Rehab 1985: 12: 81-90.

32. Prayitno $S$, Addy $M$. An in vitro study of factors affecting the development of staining associated with the use of chlorhexidine. J Periodont Res 1979; 14: 397-402.

33. Binney $A$, Addy $M$, Newcombe $R G$. The effect of a number of commercial mouthrinses compared with toothpaste on plaque regrowth. J Periodont 1992; 63: 839-842.

34. Addy M, Wade W G, Jenkins S, Goodfield S. Comparison of two commercially available chlorhexidine mouthrinses: I. Staining and antimicrobial effects in vitro. Clin Prevent Dent 1989: 11: 10-14.

35. Barkvoll P, Rölla G, Svenden A K. Interaction between chlorhexidine gluconate and sodium lauryl sulphate in vivo. J Clin Periodontol 1989; 16: 593-595. 